Original Research Paper

\title{
Presents Some Aspects Related to the Atom and Atomic Electrons, Necessary in Understanding Chemical Bonds and Nanotechnologies
}

\author{
Florian Ion Tiberiu Petrescu \\ IFToMM, ARoTMM, Bucharest Polytechnic University, Bucharest, Romania
}

Article history

Received: 16-03-2020

Revised: 28-04-2020

Accepted: 23-05-2020

Email: fitpetrescu@gmail.com

\begin{abstract}
The paper presents briefly some aspects regarding the atom and its electrons, these being absolutely necessary for understanding the molecular bonds and future nanotechnologies. It is briefly presented how to determine the energies of the atomic electrons, their speeds of movement in the atomic orbit but also of rotation around a proper axis of the atomic electron, the kinetic energies of the atomic electron at the orbital displacement and at its own rotation around its own axis, as well as the dimensions of the orbital electron depending on its velocity of movement in the atomic orbit. All these aspects presented in the paper will be able to serve in the future to a better understanding of the molecular bonds between atoms, connections made through atomic electrons, but also to the way in which new atomic bonds can be made to change the matter properties and start new atomic and molecular structures for the obvious purpose of creating new nanotechnologies capable of making more interesting links with various new properties needed in various engineering uses.
\end{abstract}

Keywords: Matter, Structure, Dimensions, Nuclear Energy, Fusion, Elementary Particle Dynamics, Condensed Matter, Atomic Electron, Electron Energy, Electron Velocity

\section{Introduction}

The Rutherford atomic model, developed by Ernest Rutherford in 1911, is the first planetary model of the atom. According to this model, the atom consists of the nucleus, in which the positive charge is concentrated and electrons that rotate around the nucleus in circular orbits, like the planets in the Solar System (Fig. 1), (Bernard, 1998; Rutherford, 1911).

The model was developed from experiments conducted by Hans Geiger and Ernest Marsden in 1909. They studied, under the guidance of Ernest Rutherford, the scattering of $\alpha$ particles as they pass through a thin sheet of gold. According to the atomic model developed by Thomson, the particles had to be deflected by a few degrees when passing through the metal due to electrostatic forces. It was found, however, that some of them were deviated by angles greater than $90^{\circ}$ or even $180^{\circ}$ (Rutherford, 1911). This was explained by the existence of a non-uniform distribution of electric charge inside the atom. Based on the observations made,
Rutherford proposed a new model in which the positive charge was concentrated in the center of the atom and the electrons orbiting around it (Halliday and Robert, 1966).

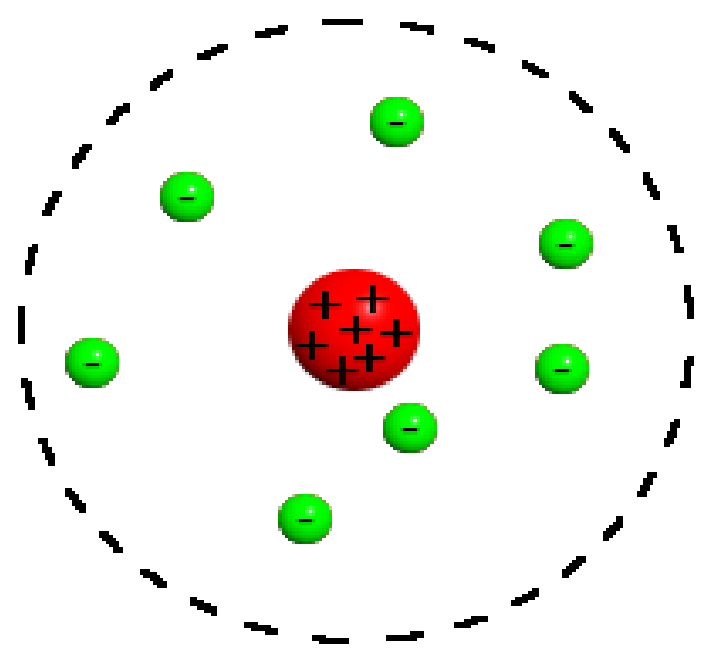

Fig. 1: The Rutherford atomic model, 1911 
The new model introduced the notion of the nucleus, without calling it that. Rutherford referred, in his 1911 paper, to a concentration of positive electric charge (Bernard, 1998):

"It is considered the passage of a high-velocity particle through an atom having a central positive charge $\mathrm{N}$ e, offset by the charge of $\mathrm{N}$ electrons."

He estimated, for energy reasons, that for the gold atom, it would have a radius of at most $3.4 \times 10-14 \mathrm{~m}$ (the current value is about one-fifth of it). The size of the radius of the gold atom was estimated at 10-10 m, almost 3000 times larger than that of the nucleus.

Rutherford assumed that the size of the positive charge would be proportional to the atomic mass expressed in atomic units, having half its value. It obtained an atomic mass of 196 for gold (compared to 197 , the current value). He did not correlate with the atomic number $\mathrm{Z}$, estimating the value of the charge at 98 e, compared to 79, where e represents the charge of the electron (Bernard, 1998).

The model proposed by Rutherford describes the nucleus but does not attribute any structure to the orbits of the electrons. However, the paper mentions Hantaro Nagaoka's Saturnian model, in which electrons are arranged on rings.

The main disadvantage of the model was that it did not explain the stability of the atom. Being developed in accordance with classical theories, it assumed that electrons in a circular motion, so accelerated, constantly emit electromagnetic radiation losing energy. Therefore, over time, the electrons would no longer have enough energy to stay in orbit and would "fall" on the nucleus.

Also, the frequency of the emitted radiation should have taken any value, depending on the frequency of electrons in the atom, which is refuted by experimental studies on spectral series.

Rutherford's model introduced the idea of an atom structure and the existence of component particles, as well as the possibility of their separation. Representing the starting point of the Bohr model, it led to the separation of two fields, nuclear physics, which studies the nucleus and atomic physics, which studies the electronic structure of the atom (Berryman, 2008).

Despite the shortcomings, the descriptive nature of the model allowed its use as a symbol of the atom and atomic energy (Halliday and Robert, 1966).

The Bohr atomic model is the first quantum model of the atom and was introduced in 1913 by the Danish physicist Niels Bohr. This model takes Ernest Rutherford's planetary model and applies the quantum theory to it. Although the hypotheses introduced by Bohr are quantum in nature, the actual calculations of atom-specific quantities are purely classical, the model being, in fact, semi-quantum. Bohr's model is applicable to hydrogen ions $(\mathrm{He}+, \mathrm{Li}+2, \mathrm{Be}+3$, etc., i.e., ions that have a single electron in the effective charge field of the nucleus).

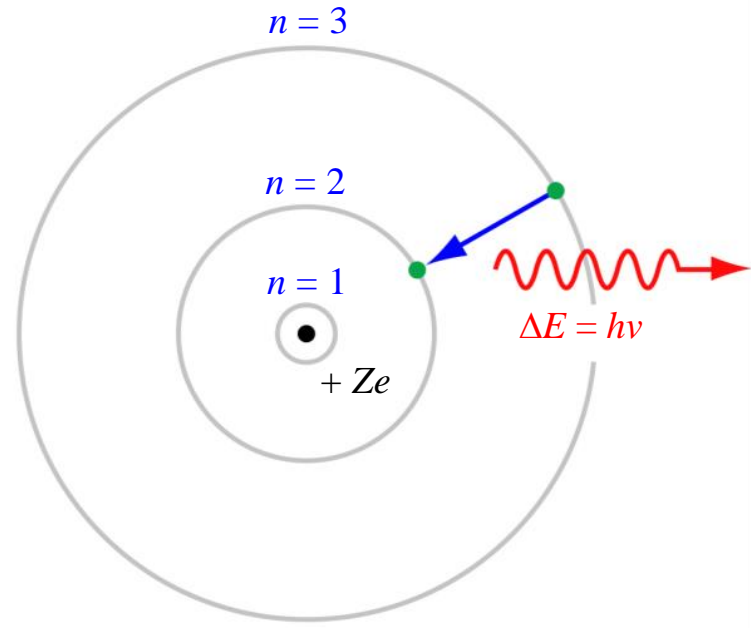

Fig. 2: Bohr atomic model for the hydrogen atom

Improving the Rutherford model is largely a quantum physical interpretation of it. The key success of the model is to explain the Rydberg formula for the spectral emission lines of atomic hydrogen. While the Rydberg formula had been known experimentally, it did not have a theoretical basis until the introduction of the Bohr model. The Bohr model not only explains the reason for the structure of the Rydberg formula, but also the justification of its empirical results in terms of fundamental physical constants (Fig. 2).

The Bohr model is a relatively primitive model of the hydrogen atom, compared to the valence shell atom. As a theory, it can be derived as a first-order approximation of the hydrogen atom using broader and more accurate quantum mechanics and can thus be considered an outdated scientific theory. However, due to its simplicity and correct results for selected systems, the Bohr model is still commonly taught as an introduction to quantum mechanics or energy level diagrams before moving on to a more precise one, the atom of valence shells. A similar model was originally proposed by Arthur Erich Haas in 1910 but was rejected. The quantum theory of the period between Planck's discovery of the quantum (1900) and the emergence of mature quantum mechanics (1925) is often referred to as the old quantum theory, Niels Bohr (1913).

In the early 20th century, Ernest Rutherford's experiments determined that atoms consist of a diffuse cloud of negatively charged electrons surrounding a small, dense, positively charged nucleus. Given these experimental data, Rutherford naturally considered an atom as a planetary model, the 1911 Rutherford model electrons orbiting a solar nucleus - however, the atom as a planetary model had a technical difficulty. The laws of classical mechanics (i.e., the Larmor formula) predict that the electron will release electromagnetic radiation as it orbits the nucleus. Because the electron would lose 
energy, it would be drawn in a spiral rapidly inward, collapsing into the nucleus within 16 picoseconds. This atomic model is disastrous because it predicts that all atoms are unstable (Berryman, 2008).

Also, as electrons spiral inside, the emission would increase rapidly in frequency, as the orbit became smaller and faster. This would produce a continuous diffusion, in frequency, of the electromagnetic radiation. However, nineteenth-century experiments with electric discharges showed that atoms would emit light (i.e., electromagnetic radiation) only at certain discrete frequencies (Halliday and Robert, 1966).

To overcome this difficulty, Niels Bohr proposed, in 1913, what is now called Bohr's model of the atom. He suggested that electrons could only have certain classical motions (Schrödinger, 1926):

The electrons in the atoms orbit the nucleus.

Electrons can only orbit stably, without radiating, on certain orbits (called by Bohr "stationary orbits") at a certain discrete set of distances from the nucleus. These orbits are associated with defined energies and are also called energy shells or energy levels. In these orbits, the acceleration of the electron does not result in radiation and energy loss, as required by classical electromagnets. Bohr's model of an atom is based on the quantum theory of Planck radiation (Einstein, 1905).

Electrons can only gain and lose energy by jumping from one allowed orbit to another, absorbing or emitting electromagnetic radiation with a frequency $v$ determined by the energy difference of the levels in the Planck relation:

$$
\Delta E=E 2-E 1=h v
$$

where, $h$ is the Planck's constant. The frequency of radiation emitted in the orbit of period $T$ is the same as in classical mechanics; it is the reciprocal of the classical orbit period:

$$
v=1 / T \text {. }
$$

Once an electron is in the smallest orbit, it cannot approach the proton. Starting from the quantum rule of angular momentum, Bohr was able to calculate the energies of the allowed orbits of the hydrogen atom and other hydrogen-like atoms and ions (Fig. 3) Niels Bohr (1913).

Other points are:

As in Einstein's theory of the photoelectric effect, Bohr's formula assumes that during a quantum leap a discrete amount of energy is emitted. However, unlike Einstein, Bohr stuck to the classical Maxwell theory of the electromagnetic field (Einstein, 1905). The quantification of the electromagnetic field was explained by the discrepancy of atomic energy levels; Bohr did not believe in the existence of photons (Niels Bohr, 1913; Einstein, 1905; Schrödinger, 1926).

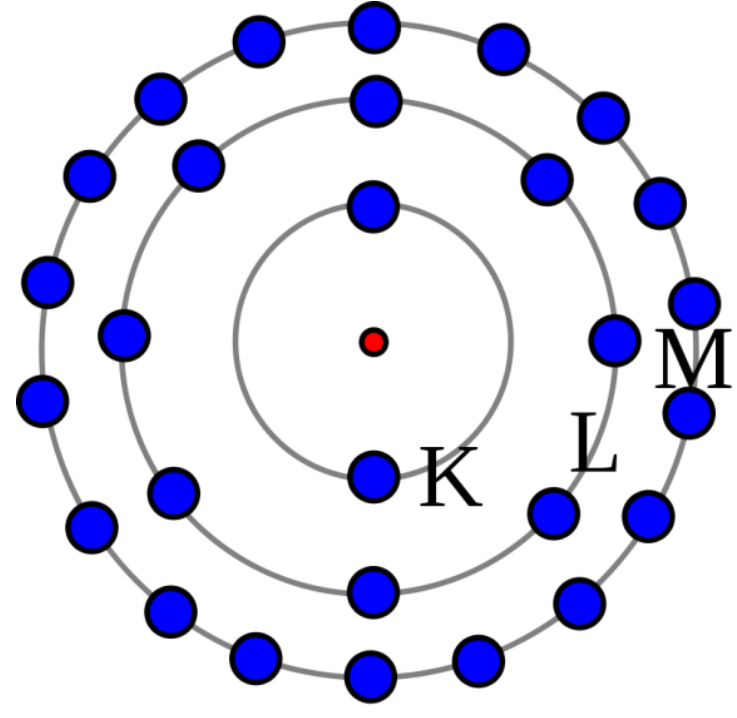

Fig. 3: Bohr model with the maximum number of electrons per shell with coatings marked in X-ray notation

According to Maxwell's theory, the frequency $v$ of classical radiation is equal to the rotation frequency vrot of the electron in its orbit, with harmonics at integers of this frequency. This result is obtained from the Bohr model for jumps between the energy levels En and En-k when k is much smaller than $n$. These jumps reproduce the frequency of the ka orbital harmonic n. For sufficiently large values of $n$ (so-called Rydberg states), the two orbits involved in the emission process have almost the same rotational frequency, so the classical orbital frequency is not ambiguous. But for small $\mathrm{n}$ (or $\mathrm{k}$ ), the radiation frequency does not have a clear classical interpretation. This marks the birth of the principle of correspondence, requiring that quantum theory agrees with classical theory only within the limits of large quantum numbers, (Halliday and Robert, 1966; Niels Bohr, 1913).

The Bohr-Kramers-Slater theory (BKS theory) is a failed attempt to extend the Bohr model, which violates the conservation of energy and momentum in quantum leaps, keeping the conservation laws only on average.

Bohr's condition, according to which the angular momentum is an integer multiple of $\hbar$, was reinterpreted in 1924 by de Broglie as a standing wave condition: The electron is described by a wave and an integer number of wavelengths must match along the circumference of the electron orbit:

$$
n \lambda=2 \pi r
$$

Bohr described an angular momentum of the electron orbit as $1 / 2 \mathrm{~h}$ while de Broglie's wavelength $\lambda=h / p$ described $\mathrm{h}$ divided by the electron pulse. In 1913, however, Bohr justified his rule by resorting to the principle of correspondence, without offering any 
interpretation of the waves. In 1913, the undulating behavior of matter particles, such as electrons (i.e., waves of matter), was not suspected, Niels Bohr (1913).

In 1925, a new type of mechanics was proposed, quantum mechanics, in which Bohr's electron model moving in quantized orbits was extended to a more precise model of electron motion. The new theory was proposed by Werner Heisenberg (Einstein, 1905). Another form of the same theory, wave mechanics, was discovered independently by the Austrian physicist Erwin Schrödinger by different reasoning. Schrödinger used Broglie's matter waves but sought wave solutions with a three-dimensional wave equation that described electrons that were forced to move around the nucleus of a hydrogen atom, being held back by the potential of the positive nuclear charge, (Schrödinger, 1926).

\section{Materials and Methods}

The electron is a fundamental subatomic particle with a negative electric charge, being symbolized e-. It is a type of spin $1 / 2$ lepton that participates in electromagnetic interactions, its mass being about $1 / 1836$ of that of the proton. Together with the atomic nucleus, the electrons constitute the atom. Their interaction with adjacent nuclei is the main cause of the chemical bonds, the valence electrons being the ones participating in the formation of these bonds (Halliday and Robert, 1966).

The name of the electron comes from the Greek word amber, $\eta \dot{\lambda} \varepsilon \kappa \tau \rho \circ \mathrm{v}$. This material has played an essential role in the discovery of electrical phenomena. The ancient Greeks knew, for example, that if a piece of amber is rubbed with a piece of fur, it causes an electrical charge on its surface, which can then create a spark when brought near a ground-bound object.

The electron carries a negative electrical charge and is considered as an elementary (quantum) unit of electrical charge whose value results from Faraday's law of electrolysis. The consideration of the electron as an elementary particle carrying a negative electrical charge was supported by the Irish physicist George Johnstone Stoney in 1874, who also invented the term electron in 1894 (Stoney, 1894).

The discovery of the electron as a subatomic particle was made in 1897 by J.J. Thomson at Cavendish Laboratory, Cambridge University while studying cathode-ray emitting tubes. A cathode ray generator tube is a sealed and vacuum glass cylinder, in which two electrodes are separated at a certain distance. When a potential difference between electrodes is applied, cathode radiation (rays) is generated which causes the tube to glow. Through this experiment, Thomson discovered that cathodic radiation can be deflected by both an electric field and a magnetic field. He deduced that these rays, believed to be where they were, were rather negatively charged particles, which he called "corpuscles" (electrons). He measured their electric charge-to-mass ratio and found it to be over a thousand times smaller than the same ratio for a hydrogen ion, suggesting that they were either very electrically charged or had a very small mass. Subsequent experiments of other scientists confirmed the latter conclusion. The proportion of the electric charge mass was also independent of the cathode material and the gas in the vacuum tube. It made Thomson realize that they are universal among all other materials.

The electron load was carefully measured by R. A. Millikan in his experiment called the oil drop in 1909.

The law of periodicity states that the chemical properties of the elements are repeated periodically and is the basis of the periodic table of the elements. The law itself was initially explained by the atomic mass of the element. Although the periodic table of the elements presents some anomalies, efforts have been made to explain them as best as possible. In 1913, Henry Moseley introduced the concept of atomic number and explained the law of periodicity from the perspective of the number of protons of each element. In the same year, Niels Bohr demonstrated that electrons are actually the basis of the table. In 1916, Gilbert Newton Lewis explained the chemical reaction of the elements through electronic interactions (Halliday and Robert, 1966).

The existence of spin moments at the electron is correlated with its geometrical structure models (electron radius). This is a non-issue that is undecided so far. Some models that admit the electron as a dot (zero radius) assume that the spin moments are due to the Zitterbewegung concept generated by the FoldyWuithuisen transformation. The proton-electron structural model requires the existence of an attractive force that counteracts the Klein paradox resulting from the indeterminacy relation.

The electron is relatively stable in solvents as ammonia, as salts of alkali metals but also in aqueous alkaline solutions (Baxendale, 1964).

The electron belongs to the class of subatomic particles called leptons, which are believed to be fundamental particles. The classical electron beam can be calculated from relativistic considerations.

Like almost all particles, electrons can behave like waves. This is called particle-wave dualism, better known as the complementarity proposed by Niels Bohr and can be demonstrated by the double-slit experiment.

The antiparticle of the electron is the positron which is a particle with a positive electrical charge. The one who discovered the positron, Carl D. Anderson proposed that standard electrons be called negatrons and that the generic term electron be used to describe both the positive and negative charge variants. Today, this use is rarely encountered.

The term atomic orbital is a notion in quantum physics used to describe the atom. It is a wave function 
that probably describes the position of electrons around the nucleus.

The occupation of orbitals with electrons is based on the following rules:

\section{The Principle of Stability}

In the fundamental state of the atom, the electrons tend to occupy the orbitals characterized by the lowest energy, from the nucleus $(\mathrm{n}=1)$ to the outside.

\section{Pauli's Exclusion Principle}

Two electrons belonging to the same atom cannot be characterized by the same quantum numbers $n, 1, m$ and s. the quantum number of spin. Since this number has only two values, $+1 / 2$ and $-1 / 2$, it turns out that only two electrons can be placed on one orbital (which differs in value from $s$ ).

\section{Hund's Rule}

Electrons are distributed in the electronic shell so that the number of parallel spin electrons (uncoupled) on orbitals with the same energy (have the same quantum numbers $\mathrm{n}$ and $\mathrm{l}$ ) is as large as possible. The rule shows that in the case of degenerate orbitals from the same layer (they have equal energies) the placement of electrons is done by occupying each degenerate orbital with one electron and then, if there are still electrons, their spin coupling is performed.

In quantum chemistry, a molecular orbital (OM) is a wave function that describes the position of electrons within a molecule. This mathematical function can help determine some properties of the molecule, such as the probability of finding the electron in a particular region, diamagnetism and paramagnetism. The term orbital was introduced by Mulliken (1932).

In some papers (Halliday and Robert, 1966; Petrescu, 2012a; 2012b; 2012c; 2014; 2018; 2019; Petrescu et al., 2017a; 2017b; 2017c; 2017d; Petrescu and Petrescu, 2018; 2019) it has been shown that dimensions of atoms and subatomic particles are measured at approximately average values and for low displacement rates of the particles. Dynamically, the dimensions of these particles vary greatly with the variation in their displacement speed. The same thing happens with the energies of these permanently moving particles.

The paper presents briefly some aspects regarding the atom and its electrons, these being absolutely necessary for understanding the molecular bonds and future nanotechnologies. It is briefly presented how to determine the energies of the atomic electrons, their speeds of movement in the atomic orbit but also of rotation around a proper axis of the atomic electron, the kinetic energies of the atomic electron at the orbital displacement and at its own rotation around its own axis, as well as the dimensions of the orbital electron depending on its velocity of movement in the atomic orbit. The calculations presented in this paper refer strictly to an atomic electron, they were determined by the authors many years ago when several possibilities for quantifying atomic electrons were established (Petrescu, 2012c).

The kinetic energy of an atomic electron can be expressed directly in the formula (1) and as electrostatic energy (Colombian) in the equation (2) and by their equalization it is deduced the form (3) that expresses the velocity of the atomic electron in orbit depending on the mass of the electron $\mathrm{m}$, the charge that is, the radius of the orbit of the electron $r$, eps0 (vacuum permittivity, permittivity of free space or electric constant or the distributed capacitance of the vacuum, is an ideal, baseline physical constant, which is the value of the absolute dielectric permittivity of classical vacuum) and the atomic number $Z$ :

$$
\begin{aligned}
E_{C} & =\frac{1}{2} m \cdot v^{2} \\
E_{C} & =\frac{1}{8} \frac{Z \cdot e^{2}}{\pi \cdot \varepsilon_{0} \cdot r} \\
v^{2} & =\frac{1}{4} \frac{Z \cdot e^{2}}{\pi \cdot \varepsilon_{0} \cdot r \cdot m}
\end{aligned}
$$

Next we write the relation (4) of Niels Bohr regarding the quantized orbit radius (distance between orbital electron and the centre of its atom) of the electron on its atomic orbit, where $\mathrm{h}$ is the Planck's constant and $\mathrm{n}$ is Bohr's quantum number:

$r=\frac{n^{2} \cdot \varepsilon_{0} \cdot h^{2}}{\pi \cdot e^{2} \cdot Z \cdot m}$

The value of the quantized radius from Equation (4) is introduced in the relation (3) and is thus obtained for the velocity (squared) of the electron in the atomic orbit its quantified expression (5).

$v^{2}=\frac{1}{4} \frac{Z^{2} \cdot e^{4}}{\varepsilon_{0}^{2} \cdot h^{2} \cdot n^{2}}$

Any elementary particle being in a rectilinear motion moving with velocity $\mathrm{v}$ also executes a rotational motion around the main axis with an angular velocity $\omega$ (Fig. 4). And the electron, in general, will have these two types of motion, linear and rotational about its own axis. In fact, the motions are completely valid also for an atomic orbital electron. 


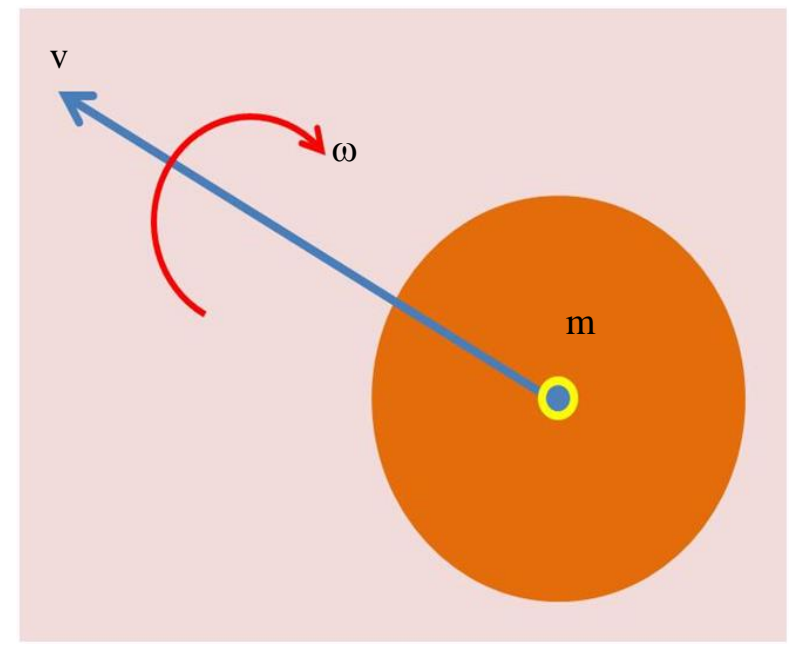

Fig. 4: The two motions of an elementary particle

There is a permanent connection between the two types of movements, so the linear and rotational motions (of the elementary particle, in our case the orbital electron) are (in other words) dependent on each other. The rotational kinetic energy of a subatomic particle (in our case the orbital electron) can be determined by the relationship (6), where $c$ is the light velocity, $h$ represents the Plank constant, $v$ is the velocity of the particle (in our case the orbital electron) in its linear movement and $\mathrm{m}$ its mass in linear movement (Petrescu, 2019; Petrescu and Petrescu, 2019):

$\omega=\frac{2 \pi \cdot c}{h} \cdot m \cdot v$

You can immediately get the square of the angular velocity of the particle, in our case the orbital electron (7):

$\omega^{2}=\frac{4 \pi^{2} \cdot c^{2}}{h^{2}} \cdot m^{2} \cdot v^{2}$

The mass at the rotation movement of the particle, in our case the orbital electron (considered by the spherical shape) is determined by the relationship (8), (Petrescu, 2019; Petrescu and Petrescu, 2019):

$J=\frac{2}{5} \cdot m \cdot R^{2}$

The kinetic energy to the particle rotation, in our case the orbital electron, is obtained with relation (9) (Petrescu, 2019; Petrescu and Petrescu, 2019):

$$
\begin{aligned}
& E_{c \omega}=\frac{1}{2} \cdot J \cdot \omega^{2} \\
& =\frac{1}{2} \cdot \frac{2}{5} \cdot m \cdot R^{2} \cdot \frac{4 \pi^{2} \cdot c^{2} \cdot m^{2} \cdot v^{2}}{h^{2}} \\
& \frac{4}{5} \frac{\pi^{2} \cdot c^{2}}{h^{2}} \cdot m^{3} \cdot v^{2} R^{2}
\end{aligned}
$$

The particle, in our case the orbital electron, radius to the square can be calculated with the original relation (10) (Petrescu, 2019; Petrescu and Petrescu, 2019). Instead of the mass of the orbital electron, its resting mass was introduced given the known Lorenz relation:

$R^{2}=\frac{5}{4} \cdot \frac{h^{2}}{\pi^{2} \cdot c^{2}} \cdot \frac{\left(1-\frac{v^{2}}{c^{2}}\right)\left(c^{2}-c \sqrt{c^{2}-v^{2}}-\frac{1}{2} v^{2}\right)}{m_{0}^{2} \cdot v^{2}}$

With the expression of the radius of the relationship (10), the kinetic energy at the rotation of particle, in our case the orbital electron, from the relationship (9) gets the form (11). Instead of the mass of the orbital electron, its resting mass was introduced given the known Lorenz relation (12):

$$
\begin{aligned}
& E_{c \omega}=m \cdot\left(c^{2}-c \sqrt{c^{2}-v^{2}}-\frac{1}{2} v^{2}\right) \\
& =m_{0} \cdot \frac{c^{2}-c \sqrt{c^{2}-v^{2}}-\frac{1}{2} v^{2}}{\sqrt{1-\frac{v^{2}}{c^{2}}}}
\end{aligned}
$$

$m=\frac{m_{0}}{\sqrt{1-\frac{v^{2}}{c^{2}}}}$

It can be seen from the relationship (6) that the kinetic energy of rotation of the particle, in our case the orbital electron, represents a percentage of its total energy. It can also be expressed as another percentage of its resting energy (relationship 13):

$E_{c \omega}=m_{0} \cdot c^{2} \cdot \frac{1-\sqrt{1-\beta^{2}}-\frac{1}{2} \beta^{2}}{\sqrt{1-\beta^{2}}} ;$ where $\beta=\frac{v}{c}$

Using the relationships (5) and (10) one can obtain the expressions (14) that define the square of the radius of a quantum electron located on an atomic orbit and the radius resumed in the form (15): 


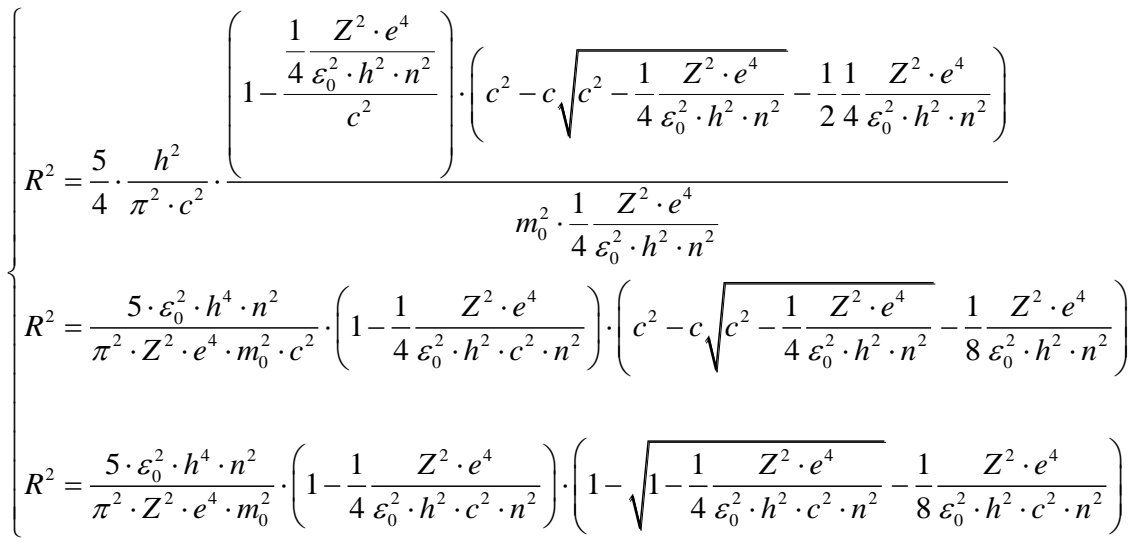

$$
\begin{aligned}
& R=\frac{\sqrt{5} \cdot \varepsilon_{0} \cdot h^{2} \cdot n}{\pi \cdot Z \cdot e^{2} \cdot m_{0}} \cdot \sqrt{1-\frac{1}{4} \frac{Z^{2} \cdot e^{4}}{\varepsilon_{0}^{2} \cdot h^{2} \cdot c^{2} \cdot n^{2}}} . \\
& \sqrt{1-\sqrt{1-\frac{1}{4} \frac{Z^{2} \cdot e^{4}}{\varepsilon_{0}^{2} \cdot h^{2} \cdot c^{2} \cdot n^{2}}}-\frac{1}{8} \frac{Z^{2} \cdot e^{4}}{\varepsilon_{0}^{2} \cdot h^{2} \cdot c^{2} \cdot n^{2}}} \\
& E_{c \omega}=m_{0} \cdot c^{2} \cdot \frac{1-\sqrt{1-\frac{1}{4} \frac{Z^{2} \cdot e^{4}}{\varepsilon_{0}^{2} \cdot h^{2} \cdot c^{2} \cdot n^{2}}}-\frac{1}{8} \frac{Z^{2} \cdot e^{4}}{\varepsilon_{0}^{2} \cdot h^{2} \cdot c^{2} \cdot n^{2}}}{\sqrt{1-\frac{1}{4} \frac{Z^{2} \cdot e^{4}}{\varepsilon_{0}^{2} \cdot h^{2} \cdot c^{2} \cdot n^{2}}}}
\end{aligned}
$$

Using the relations (7) and (5) we obtain the squared angular velocity of the orbital atomic electron (16) which is then explained as angular velocity in the form (17). Instead of the mass of the orbital electron, its resting mass was introduced given the known Lorenz relation:

$$
\begin{aligned}
& \left\{\begin{array}{l}
\left.\omega^{2}=\frac{4 \pi^{2} \cdot c^{2} \cdot m_{0}^{2}}{h^{2} \cdot\left(1-\frac{1}{\frac{4}{\varepsilon_{0}^{2} \cdot h^{2} \cdot n^{2}}} c^{2}\right.}\right) \cdot \frac{1}{4} \frac{Z^{2} \cdot e^{4}}{\varepsilon_{0}^{2} \cdot h^{2} \cdot n^{2}} \\
=\frac{\pi^{2} \cdot c^{2} \cdot m_{0}^{2}}{\left(1-\frac{1}{4} \frac{Z^{2} \cdot e^{4}}{\varepsilon_{0}^{2} \cdot h^{2} \cdot c^{2} \cdot n^{2}}\right)} \cdot \frac{Z^{2} \cdot e^{4}}{\varepsilon_{0}^{2} \cdot h^{4} \cdot n^{2}} \\
=\frac{4 \pi^{2} \cdot c^{2} \cdot m_{0}^{2}}{\left(4 \varepsilon_{0}^{2} \cdot h^{2} \cdot c^{2} \cdot n^{2}-Z^{2} \cdot e^{4}\right)} \cdot \frac{Z^{2} \cdot e^{4} \cdot c^{2}}{h^{2}}
\end{array}\right. \\
& \omega=\frac{2 \pi \cdot c \cdot m_{0}}{\sqrt{4 \varepsilon_{0}^{2} \cdot h^{2} \cdot c^{2} \cdot n^{2}-Z^{2} \cdot e^{4}}} \cdot \frac{Z \cdot e^{2} \cdot c}{h}
\end{aligned}
$$

The rotational energy of the orbital electron is expressed by the relation (18) deduced from expressions (11) and (5) and resumed in the form (19):

$$
\left\{\begin{array}{l}
E_{c \omega}=m_{0} \cdot \frac{c^{2}-c \sqrt{c^{2}-\frac{1}{4} \frac{Z^{2} \cdot e^{4}}{\varepsilon_{0}^{2} \cdot h^{2} \cdot n^{2}}}-\frac{1}{2} \frac{1}{4} \frac{Z^{2} \cdot e^{4}}{\varepsilon_{0}^{2} \cdot h^{2} \cdot n^{2}}}{\sqrt{\frac{1}{1-\frac{1}{4} \frac{Z^{2} \cdot e^{4} \cdot h^{2} \cdot n^{2}}{c^{2}}}}} \\
=m_{0} \cdot c^{2} \cdot \frac{1-\sqrt{1-\frac{1}{4} \frac{Z^{2} \cdot e^{4}}{\varepsilon_{0}^{2} \cdot h^{2} \cdot c^{2} \cdot n^{2}}}-\frac{1}{8} \frac{Z^{2} \cdot e^{4}}{\varepsilon_{0}^{2} \cdot h^{2} \cdot c^{2} \cdot n^{2}}}{\sqrt{1-\frac{1}{4} \frac{Z^{2} \cdot e^{4}}{\varepsilon_{0}^{2} \cdot h^{2} \cdot c^{2} \cdot n^{2}}}}
\end{array}\right.
$$

It can be observed that the rotational energy of the orbital electron represents a percentage of its resting energy.

\section{Results and Discussion}

The following will highlight the useful relationships for calculating some of the essential parameters of an orbital electron.

The relation (20) expresses the speed at which the orbital electron moves on the atomic orbit:

$v=\frac{1}{2} \frac{Z \cdot e^{2}}{\varepsilon_{0} \cdot h \cdot n}$

Expression (21) determines the angular velocity of the orbital electron, with which it rotates about a principal axis of its own:

$\omega=\frac{2 \pi \cdot c \cdot m_{0}}{\sqrt{4 \varepsilon_{0}^{2} \cdot h^{2} \cdot c^{2} \cdot n^{2}-Z^{2} \cdot e^{4}}} \cdot \frac{Z \cdot e^{2} \cdot c}{h}$

Expression (22) indicates the radius of the orbital electron, with which the diameter of the moving electron can be determined by doubling. It is a dynamic size, which varies according to several parameters:

$$
\begin{aligned}
& R=\frac{\sqrt{5} \cdot \varepsilon_{0} \cdot h^{2} \cdot n}{\pi \cdot Z \cdot e^{2} \cdot m_{0}} \cdot \sqrt{1-\frac{1}{4} \frac{Z^{2} \cdot e^{4}}{\varepsilon_{0}^{2} \cdot h^{2} \cdot c^{2} \cdot n^{2}}} \cdot \\
& \sqrt{1-\sqrt{1-\frac{1}{4} \frac{Z^{2} \cdot e^{4}}{\varepsilon_{0}^{2} \cdot h^{2} \cdot c^{2} \cdot n^{2}}}-\frac{1}{8} \frac{Z^{2} \cdot e^{4}}{\varepsilon_{0}^{2} \cdot h^{2} \cdot c^{2} \cdot n^{2}}}
\end{aligned}
$$

The relation (23) generates the kinetic energy of the electron moving in the atomic orbit, representing only the kinetic energy at the linear displacement in the orbit 
without taking into account the rotational energy of the electron which represents a distinct value:

$$
E_{c v}=\frac{1}{2} m_{0} \cdot c^{2} \cdot \frac{\frac{1}{4} \frac{Z^{2} \cdot e^{4}}{\varepsilon_{0}^{2} \cdot h^{2} \cdot c^{2} \cdot n^{2}}}{\sqrt{1-\frac{1}{4} \frac{Z^{2} \cdot e^{4}}{\varepsilon_{0}^{2} \cdot h^{2} \cdot c^{2} \cdot n^{2}}}}
$$

Equation (24) precisely determines the rotational energy of the orbital electron around its own axis. This energy is responsible for creating a wave associated with the quantum orbital electron particle:

$$
E_{c \omega}=m_{0} \cdot c^{2} \cdot \frac{1-\sqrt{1-\frac{1}{4} \frac{Z^{2} \cdot e^{4}}{\varepsilon_{0}^{2} \cdot h^{2} \cdot c^{2} \cdot n^{2}}}-\frac{1}{8} \frac{Z^{2} \cdot e^{4}}{\varepsilon_{0}^{2} \cdot h^{2} \cdot c^{2} \cdot n^{2}}}{\sqrt{1-\frac{1}{4} \frac{Z^{2} \cdot e^{4}}{\varepsilon_{0}^{2} \cdot h^{2} \cdot c^{2} \cdot n^{2}}}}
$$

The expression (25) of the radius of the orbital electron $r$, refers to the distance between the orbital electron and the center of the mother atom:

$$
r=\frac{n^{2} \cdot \varepsilon_{0} \cdot h^{2} \cdot \sqrt{1-\frac{1}{4} \frac{Z^{2} \cdot e^{4}}{\varepsilon_{0}^{2} \cdot h^{2} \cdot c^{2} \cdot n^{2}}}}{\pi \cdot e^{2} \cdot Z \cdot m_{0}}
$$

\section{Conclusion}

The paper presents briefly some aspects regarding the atom and its electrons, these being absolutely necessary for understanding the molecular bonds and future nanotechnologies.

It is briefly presented how to determine the energies of the atomic electrons, their speeds of movement in the atomic orbit but also of rotation around a proper axis of the atomic electron, the kinetic energies of the atomic electron at the orbital displacement and at its own rotation around its own axis, as well as the dimensions of the orbital electron depending on its velocity of movement in the atomic orbit.

The relation (20) expresses the speed at which the orbital electron moves on the atomic orbit.

Expression (21) determines the angular velocity of the orbital electron, with which it rotates about a principal axis of its own.

Expression (22) indicates the radius of the orbital electron, with which the diameter of the moving electron can be determined by doubling. It is a dynamic size, which varies according to several parameters.

The relation (23) generates the kinetic energy of the electron moving in the atomic orbit, representing only the kinetic energy at the linear displacement in the orbit without taking into account the rotational energy of the electron which represents a distinct value.

Equation (24) precisely determines the rotational energy of the orbital electron around its own axis. This energy is responsible for creating a wave associated with the quantum orbital electron particle.

The expression (25) of the radius of the orbital electron $r$, refers to the distance between the orbital electron and the center of the mother atom.

All these aspects presented in the paper will be able to serve in the future to a better understanding of the molecular bonds between atoms, connections made through atomic electrons, but also to the way in which new atomic bonds can be made to change the matter properties and start new atomic and molecular structures for the obvious purpose of creating new nanotechnologies capable of making more interesting links with various new properties needed in various engineering uses.

The paper combines the Bohr model with current quantum physics, in order to improve the atomic, hydrogen and general model and it is useful in modern nuclear physics, chemistry and nuclear energy.

\section{Acknowledgment}

The author acknowledges INIS for his research in the field.

\section{Ethics}

This article is original and contains unpublished material. Author declares that are not ethical issues and no conflict of interest that may arise after the publication of this manuscript.

\section{References}

Baxendale, J.H., 1964. Effects of oxygen and $\mathrm{pH}$ in the radiation chemistry. Radiat. Res. Supplement, 4: 114-138.

Bernard, P., 1998. The Atom in the History of Human Thought. 1st Edn., Oxford University Press, Oxford, England, ISBN 978-0-19-515040-7.

Berryman, S., 2008. Ancient Atomism. In: Stanford Encyclopedia of Philosophy, Zalta E.N. (Ed.).

Niels Bohr, 1913. On the constitution of atoms and molecules, Part I. Philosophical Magazine, 26: 1-24. DOI: $10.1080 / 14786441308634955$

Einstein, A., 1905. Über die von der molekularkinetischen theorie der wärme geforderte bewegung von in ruhenden flüssigkeiten suspendierten teilchen. Annalen Physik, 322: 549-560. DOI: 10.1002/andp.19053220806

Halliday, D. and R. Robert, 1966. Physics, Part II. 1st Edn., John Wiley and Sons, Inc., New York. 
Mulliken, R.S., 1932. Electronic structures of polyatomic molecules and valence. II. General considerations. Phys. Rev., 41: 49-71.

DOI: 10.1103/PhysRev.41.49

Petrescu, F.I.T., 2014. Nuclear fusion. Infinite Energy, 20: 44-47.

Petrescu, F.I.T., 2012a. Cold Nuclear Fusion. 1st Edn., BoD - Books on Demand, ISBN-10: 3848228521, pp: 102.

Petrescu, F.I.T., 2012b. Cold Nuclear Fusion. 1st Edn., Create Space Publisher, USA, ISBN-13: 978-14782-3426-5, pp: 80.

Petrescu, F.I.T., 2012c. A New Atomic Model. Books On Demand, ISBN-13: 978-3848218943, pp: 62.

Petrescu, R.V., R. Aversa, S. Li, R. Bucinell and S. Kosaitis et al., 2017a. Electron dimensions. Am. J. Eng. Applied Sci., 10: 584-602. DOI: 10.3844/ajeassp.2017.584.602

Petrescu, R.V., R. Aversa, S. Kozaitis, A. Apicella and F.I.T. Petrescu, 2017b. Deuteron dimensions. Am. J. Eng. Applied Sci., 10: 649-654.

DOI: 10.3844/ajeassp.2017.649.654

Petrescu, R.V., R. Aversa, S. Kozaitis, A. Apicella and F.I.T. Petrescu, 2017c. Some proposed solutions to achieve nuclear fusion. Am. J. Eng. Applied Sci., 10: 703-708. DOI: 10.3844/ajeassp.2017.703.708

Petrescu, R.V., R. Aversa, S. Kozaitis, A. Apicella and F.I.T. Petrescu, 2017d. Some basic reactions in nuclear fusion. Am. J. Eng. Applied Sci., 10: 709-716. DOI: 10.3844/ajeassp.2017.709.716

Petrescu, F.I.T., 2019. About the nuclear particles' structure and dimensions. Comp. Part. Mech., 6: 191-194. DOI: 10.1007/s40571-018- 0206-7

Petrescu, F.I.T., 2018. About the triton structure. Am. J. Eng. Applied Sci., 11: 1293-1297.

DOI: 10.3844/ajeassp.2018.1293.1297

Petrescu, N. and F. Petrescu, 2018. Elementary structure of matter can be studied with new quantum computers. Am. J. Eng. Applied Sci., 11: 1062-1075.

DOI: 10.3844/ajeassp.2018.1062.1075

Petrescu, F.I.T. and R.V.V., Petrescu, 2019. Nuclear hydrogen structure and dimensions. Int. J. Hydrogen Energy, 44: 10833-10837.

DOI: $10.1016 /$ j.ijhydene.2019.02.140

Rutherford, E., 1911. The scattering of $\alpha$ and $\beta$ particles by matter and the structure of the atom. Philosophical Magazine, 21: 669-669.

DOI: $10.1080 / 14786435.2011 .617037$

Schrödinger, E., 1926. Quantisation as an eigenvalue problem. Annalen Physik, 81: 109-139.

DOI: $10.1002 /$ andp. 19263861802

Stoney, G.J., 1894. XLIX. Of the "electron," or atom of electricity. London Edinburgh Dublin Philosophical Magazine J. Sci., 38: 418-420.

DOI: $10.1080 / 14786449408620653$

\section{Nomenclature}

q

$\mathrm{c}$

$\Rightarrow$ The Planck constant: $\mathrm{h}=6.626 \mathrm{E}-34$ [Js]

$\Rightarrow$ Electrical elementary load: qe $=-1.6021$ E-19[C] qp = +1.6021 E-19[C]

$=$ The light speed in vacuum: $\mathrm{c}=$ $2.997925 \mathrm{E}+08[\mathrm{~m} / \mathrm{s}]$

The permissive constant (the permittivity):

$\varepsilon_{0}=8.85418 \cdot 10^{-12}\left[\frac{C^{2}}{N \cdot m^{2}}\right]$

$\mathrm{n} \quad=$ The principal quantum number (the Bohr quantum number);

$\mathrm{Z}=$ The number of protons from the atomic nucleus (the atomic number);

$\mathrm{m} 0[\mathrm{~kg}] \quad \Rightarrow$ The rest mass of one particle

m0electron $=9.11 \mathrm{E}-31[\mathrm{~kg}]$

m0proton $=1.672621898(21) \mathrm{E}-27[\mathrm{~kg}]$

m0neutron $=1.674927471(21) \mathrm{E}-27[\mathrm{~kg}]$

m0deuteron $=3.34449 \mathrm{E}-27[\mathrm{~kg}]$

m0triton $=5.00827 \mathrm{E}-27[\mathrm{~kg}]$

\section{Sources of Figures}

Fig. 1

https://ro.wikipedia.org/wiki/Modelul_atomic_Ruther ford\#/media/Fi\%C8\%99ier:Rutherfordsches_Atommode 11.png

Fig. 2

https://ro.wikipedia.org/wiki/Modelul_atomic_Bohr\#/ media/Fi\%C8\%99ier:Bohr_atom_model.svg

Fig. 3

https://ro.wikipedia.org/wiki/Modelul_atomic_Bohr\#/ media/Fi\%C8\%99ier:Atome_bohr_couches_electronique s_KLM.svg

Fig. 4

Drawn by author 